\title{
Efficacy and safety of vedolizumab in ulcerative colitis in patients from Asian countries in the GEMINI 1 study
}

\author{
Choon Jin Ooi ${ }^{1}$, Ida Normiha Hilmi ${ }^{2}$, Hyo-Jong $\mathrm{Kim}^{3}$, Umesh Jalihal ${ }^{4}$, Deng-Chyang $\mathrm{Wu}^{5}$, Dirk Demuth ${ }^{6}$, \\ Dirk Lindner ${ }^{7}$, Shashi Adsul ${ }^{7}$ \\ ${ }^{1}$ Duke-NUS Medical School, Gleneagles Medical Centre, Singapore; ${ }^{2}$ University Malaya Medical Centre, Kuala Lumpur, Malaysia; ${ }^{3}$ Kyung Hee \\ University School of Medicine, Seoul, Korea; ${ }^{4}$ M. S. Ramaiah Medical College and Hospital, Bangalore, India; ${ }^{5}$ Kaohsiung Medical University \\ Hospital, Kaohsiung Medical University, Kaohsiung, Taiwan; ${ }^{6}$ Takeda Pharmaceutical International AG, Singapore; ${ }^{7}$ Takeda Pharmaceutical \\ International AG, Zurich, Switzerland
}

Background/Aims: The efficacy and safety of vedolizumab in moderate to severely active ulcerative colitis (UC) have been demonstrated in the GEMINI 1 study (NCT00783718). This post-hoc exploratory analysis sought to establish the efficacy and safety of vedolizumab in a subgroup of patients from Asian countries with UC from GEMINI 1. Methods: Efficacy outcomes of interest were clinical response, clinical remission and mucosal healing at week 6 (induction phase); and clinical remission, durable clinical response, durable clinical remission, mucosal healing and glucocorticoid-free remission at week 52 (maintenance phase). Differences in outcome rates between vedolizumab and placebo in Asian countries (Hong Kong, India, Malaysia, Singapore, South Korea, and Taiwan) were assessed using descriptive analyses, and efficacy and safety compared between Asian and non-Asian countries. Results: During induction, in Asian countries $(\mathrm{n}=58)$, clinical response rates at week 6 with vedolizumab and placebo were $55.2 \%$ and $24.1 \%$, respectively (difference $31.0 \%$; $95 \%$ confidence interval: $7.2 \%-54.9 \%$ ). In non-Asian countries $(n=316)$, response rates at week 6 with vedolizumab and placebo were $45.9 \%$ and $25.8 \%$, respectively. During maintenance, in Asian countries, clinical remission rates at 52 weeks with vedolizumab administered every 8 weeks, vedolizumab administered every 4 weeks and placebo were $9.1 \%, 36.8 \%$, and $31.6 \%$, respectively; corresponding rates for mucosal healing were $45.5 \%, 47.4 \%$, and $47.4 \%$, respectively. Vedolizumab was well-tolerated; adverse event frequency was comparable in Asian and non-Asian countries. Conclusions: In patients from Asian countries, the efficacy and safety of vedolizumab in treatment of UC were broadly consistent with that in the overall study population. (Intest Res 2021;19:71-82)

Key Words: Vedolizumab; Colitis, ulcerative; Asia; Remission induction; Clinical efficacy

\section{INTRODUCTION}

Ulcerative colitis (UC) and Crohn's disease (CD) are together termed inflammatory bowel disease (IBD), which are chronic, relapsing, inflammatory disorders. UC is the more common type of IBD and usually affects the rectum along with some part of the colon proximal to the rectum. ${ }^{1}$ It is characterized by continuous mucosal inflammation with symptoms of bloody

Received December 16, 2019. Revised March 24, 2020. Accepted April 6, 2020. Correspondence to Choon Jin Ooi, Duke-NUS Medical School, Gleneagles Medical Centre, 6 Napier Road, \#10-02 Singapore 258499, Singapore. Tel: +65-6471-2500, Fax: +65-6471-2578, E-mail: eddyooi@duke-nus.edu.sg diarrhea and abdominal cramps. ${ }^{2}$ The incidence of UC ranges from 1.2 to 20.3 per 100,000 person-years while the prevalence ranges from 7.6 to 245 per 100,000 persons. ${ }^{2}$ The incidence and prevalence of UC are much lower in Asia than the West, but appear to be steadily increasing over the last 2 to 4 decades, with higher incidence in urban areas. ${ }^{3,4}$ In a population-based study in South Korea, the incidence of UC increased from 0.34 per 100,000 person-years in 1986-1990 to 3.08 per 100,000 person-years in 2001 to $2005 .^{5}$

Typically, a "step-up" approach is used for the management of UC. Based on disease severity, the treatment may involve the use of 5-aminosalicylic acid, glucocorticoids, immune 
modulators, biologics and/or surgery. In the last two decades, anti-tumor necrosis factor (anti-TNF) agents have played an increasing role in the management of UC. ${ }^{2}$ However, high rates of primary and secondary nonresponse, ${ }^{6}$ as well as the risk of serious infections remain important limiting factors with these drugs-the risk of opportunistic infections is doubled and that of tuberculosis (TB) infection (TB reactivation or primary infection) is increased 2.5 times with anti-TNF agents. ${ }^{7}$ An increased risk of TB infection is of particular concern in many Asian countries where TB remains a major public health problem. ${ }^{8}$

Advances in UC treatment include the development of adhesion molecule/integrin antagonists. ${ }^{2,9}$ Vedolizumab, a gutselective alpha4beta7 integrin antagonist, is approved for the treatment of moderate to severely active UC after failure with conventional therapy or anti-TNF agents. The efficacy and safety of vedolizumab in UC was demonstrated in the GEMINI 1 study (NCT00783718), ${ }^{10}$ and has also been shown to be effective in real-world clinical practice. ${ }^{11}$ Importantly, data covering more than 100,000 patient-years suggest that the risk of opportunistic infections, including TB, is lower with adhesion molecule antagonists than with anti-TNF agents. ${ }^{12}$

Most of the data on the efficacy and safety of biologics in IBD have been generated in Western countries with limited data from Asian countries. Asia-specific data are important as genetic and environmental differences may potentially influence therapeutic responses and complication rates. ${ }^{13-15}$ Therefore, there is an urgent need for more data on the efficacy and safety of biologics on Asian patients with UC.

The objective of this post-hoc exploratory analysis was to evaluate the efficacy and safety of vedolizumab in a subgroup of patients from Asian countries with UC in the GEMINI 1 study and compare it with the non-Asian subgroup from the same study.

\section{METHODS}

GEMINI 1 was a phase 3, randomized, double-blind, placebocontrolled study. The study was conducted at 211 centers from 34 countries across the world, including 6 Asian countries (Hong Kong, India, Malaysia, Singapore, South Korea, and Taiwan) and 28 non-Asian countries (Australia, Austria, Belgium, Bulgaria, Canada, Czech Republic, Denmark, Estonia, France, Germany, Greece, Hungary, Iceland, Ireland, Israel, Italy, Latvia, Netherlands, New Zealand, Norway, Poland, Russia, South Africa, Spain, Switzerland, Turkey, United Kingdom, and Unit- ed States). The protocol was approved by the institutional review board at each participating center. All patients gave written informed consent. The detailed methodology of this study has been reported earlier ${ }^{10}$ and only the key elements will be summarized here.

\section{Eligibility Criteria}

The GEMINI 1 study involved patients with active UC (Mayo clinic score of 6 to 12) between 18 to 80 years of age who either did not respond to or experienced unacceptable adverse events (AEs) with previous treatment (glucocorticoid, immunosuppressive agents or TNF antagonists). Patients previously treated with humanized monoclonal antibodies (vedolizumab, natalizumab, efalizumab, or rituximab) and those at increased risk of infection-related complications were excluded.

\section{Induction Phase (till Week 6)}

In cohort 1 , patients were randomly assigned, in a 3:2 ratio, to double-blind induction therapy with intravenous vedolizumab $300 \mathrm{mg}$ or placebo at days 1 and 15. These patients constituted the induction intent-to-treat (ITT) population. In cohort 2 , additional patients were included in an open-label vedolizumab group, with all patients in this cohort receiving the same vedolizumab induction regimen as in cohort 1 . Cohort 1 and cohort 2 patients together constituted the safety population for the induction phase. Clinical response was assessed at week 6 (defined as decrease in the Mayo Clinic score of $\geq 3$ points and a decrease of $\geq 30 \%$ from the baseline score, with a decrease of $\geq 1$ point on the rectal bleeding subscale or an absolute rectal bleeding score of 0 or 1 ).

\section{Maintenance Phase (Week 6 till Week 52)}

All patients who showed clinical response to vedolizumab at week 6 were randomly assigned to receive double-blind treatment with vedolizumab every 8 weeks (q8w), vedolizumab every 4 weeks (q4w), or placebo for up to 52 weeks. These patients constituted the maintenance ITT population.

Patients without response to vedolizumab induction therapy at week 6 were administered open-label vedolizumab 300 mg q4w and followed through week 52. Patients who received placebo during induction continued to receive placebo during the maintenance phase. Both these groups of patients (openlabel vedolizumab during maintenance and placebo in induction+maintenance), along with the maintenance ITT population, constituted the safety population for the maintenance phase. 


\section{Outcomes}

During the induction phase, the primary outcome was clinical response at week 6 (decrease in the Mayo Clinic score of $\geq 3$ points and a decrease of $\geq 30 \%$ from the baseline score, with a decrease of $\geq 1$ point on the rectal bleeding subscale or an absolute rectal bleeding score of 0 or 1 ). Secondary induction phase outcomes were clinical remission (Mayo Clinic score of $\leq 2$ and no subscore $>1$ ) and mucosal healing (Mayo endoscopic subscore of 0 or 1 ) at week 6 . During the maintenance phase, the primary outcome was clinical remission at week 52. Secondary maintenance phase outcomes were durable clinical response (response at both weeks 6 and 52), durable clinical remission (remission at both weeks 6 and 52), mucosal healing at week 52, and glucocorticoid-free remission at week 52 in patients receiving glucocorticoids at baseline (patients using oral glucocorticoids at baseline who have discontinued glucocorticoids and are in clinical remission at week 52).

\section{Statistical Analysis}

Efficacy endpoints of the induction phase (clinical response, clinical remission and mucosal healing at week 6) were summarized for the induction ITT population (patients randomized to either vedolizumab [= cohort 1] or placebo for induction); efficacy endpoints of the maintenance phase (clinical remission at week 52, durable clinical response, durable clinical remission, mucosal healing at week 52 , and glucocorticoid-free remission at week 52) were summarized for the maintenance ITT population (patients treated with vedolizumab in induction and with response at week 6 , randomized to vedolizumab $\mathrm{q} 4 \mathrm{w}$, vedolizumab q8w or placebo for maintenance). Safety data (incidence of AEs) were summarized for the induction and maintenance safety populations (including also patients treated with open-label vedolizumab in the respective phase).

Demographic and baseline characteristics were summarized using descriptive statistics. Efficacy endpoints were summarized descriptively (number and percentage of patients achieving outcome) by randomized treatment for the induction and maintenance phase; additionally, differences in rates between vedolizumab and placebo and corresponding 95\% confidence interval (CI) (using normal approximation; exact method used if counts were less than or equal to 5) for these differences were provided.

All summaries were provided for patients in the Asian countries and for patients in the non-Asian countries. In view of the small numbers of subjects and post-hoc nature of the analysis, the data are reported only descriptively, and no comparison of the treatment effect size between the Asian and non-Asian countries was conducted.

\section{RESULTS}

Fig. 1 shows the study schematic and the number of patients in each treatment arm in the GEMINI 1 study overall, as well as in the Asian and non-Asian countries. Overall, in the induction phase of the GEMINI 1 study, 374 patients in the ITT induction population were enrolled with 225 patients randomized to vedolizumab and 149 to placebo (cohort 1); separately, in the non-ITT population, 521 patients received open-label vedolizumab (cohort 2). Three hundred and seventy-three patients showed response in induction on vedolizumab and were included in the ITT maintenance population, along with 135 patients on placebo.

\section{Asian Countries Subgroup 1) Disposition}

The disposition of the Asian subgroup of the GEMINI 1 study is shown in Fig. 1. Cohort 1 consisted of 58 patients, of whom 29 patients each were randomized to vedolizumab or placebo (induction ITT population). Cohort 2 consisted of an additional 55 patients who were treated with open-label vedolizumab; these patients were included only in the safety population for induction. Forty-nine patients (58.3\%) showed response to vedolizumab at 6 weeks and were randomized in the maintenance phase-19 to vedolizumab q4w, 11 to vedolizumab 8qw, and 19 to placebo (maintenance ITT population). Thirty patients (35.7\%) failed to show response to vedolizumab at 6 weeks and received open-label vedolizumab during maintenance; 5 patients $(6.0 \%)$ who were treated with vedolizumab discontinued the study during the induction phase. Twenty-five patients who received placebo during induction continued to receive it during maintenance.

\section{2) Demography and Baseline Characteristics}

The demography and baseline characteristics of the Asian subgroup are shown in Tables 1 and 2. In the induction phase, the median duration of UC was 2.6 years (range, $0.5-10.0$ years) in the vedolizumab cohort 1 and 2.5 years (range, $0.7-16.6$ years) in the placebo group. In the vedolizumab cohort $1,72.4 \%$ of the patients had received prior treatment with glucocorticoids and/or immunomodulators, compared to $79.3 \%$ in the placebo group; none of the patients in the vedolizumab cohort 1 and $6.9 \%$ of the patients in the placebo group and had re- 


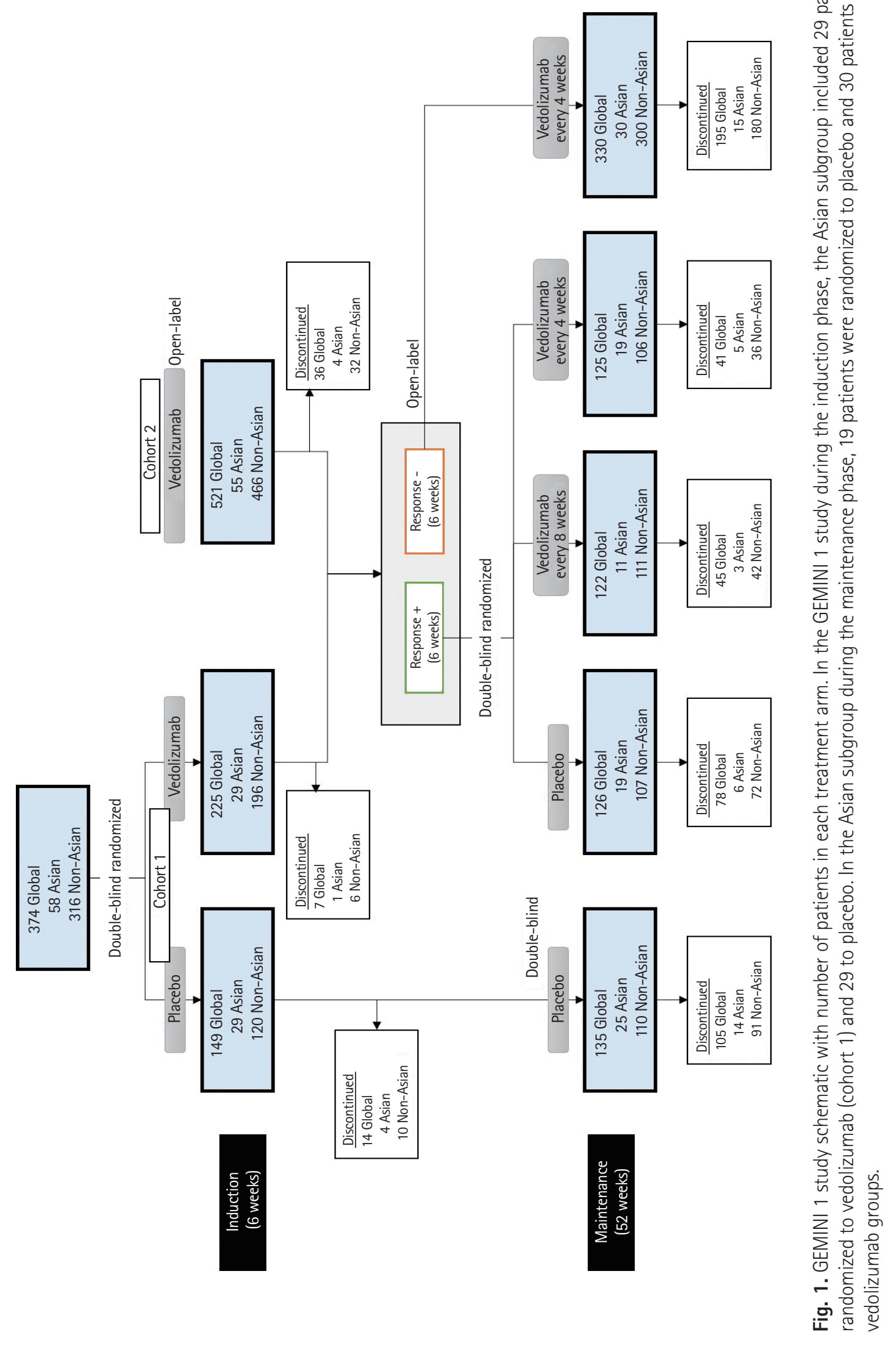


Table 1. Characteristics in the Asian Countries Subgroup of GEMINI 1 Patients: Induction Phase

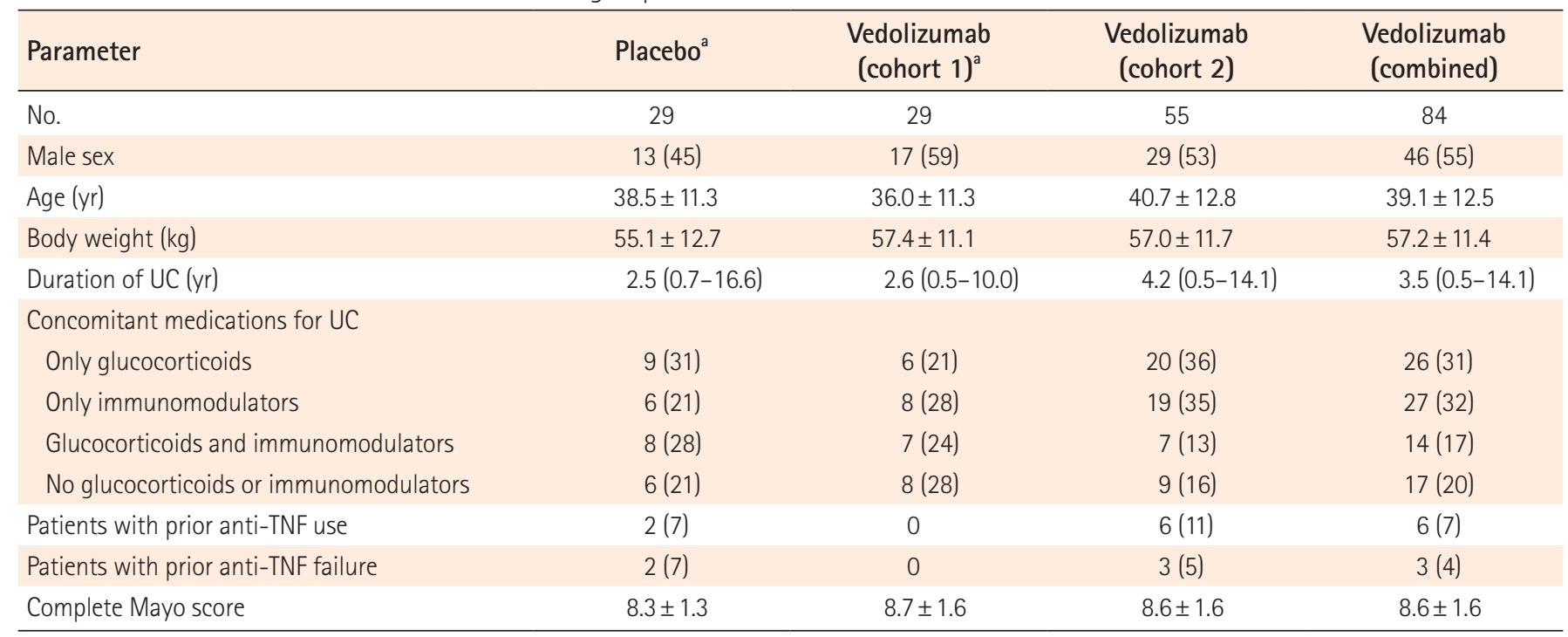

Values are presented as number (\%), mean \pm standard deviation, or median (range).

Placebo and vedolizumab (cohort 1)=the groups that were part of the double-blind induction phase (induction intent-to-treat [ITT] population); Vedolizumab (cohort 2)=additional patients were enrolled to meet the maintenance phase sample size requirements and received open-label vedolizumab (induction safety population only); Vedolizumab combined = all patients that received vedolizumab during the induction phase.

${ }^{\text {a }}$ Data for the ITT population.

UC, ulcerative colitis; TNF, tumor necrosis factor.

Table 2. Characteristics in the Asian Countries Subgroup of GEMINI 1 Patients: Maintenance Phase

\begin{tabular}{|c|c|c|c|c|c|c|c|}
\hline \multirow[b]{2}{*}{ Parameter } & \multicolumn{3}{|c|}{$\mathrm{IT}^{\mathrm{a}}$} & \multicolumn{2}{|c|}{ Non-ITT } & \multirow{2}{*}{$\begin{array}{l}\text { Placebo } \\
\text { combined }\end{array}$} & \multirow{2}{*}{$\begin{array}{c}\text { Vedolizumab } \\
\text { combined }\end{array}$} \\
\hline & Placebo & $\begin{array}{l}\text { Vedolizumab } \\
\text { q8w }\end{array}$ & $\begin{array}{l}\text { Vedolizumab } \\
\text { q4w }\end{array}$ & Placebo & $\begin{array}{l}\text { Vedolizumab } \\
\text { q4w }\end{array}$ & & \\
\hline No. & 19 & 11 & 19 & 29 & 35 & 48 & 65 \\
\hline Male sex & $10(53)$ & $5(45)$ & $9(47)$ & $13(45)$ & $22(63)$ & $23(48)$ & $36(55)$ \\
\hline Age (yr) & $35.9 \pm 13.2$ & $46.8 \pm 13.1$ & $37.9 \pm 11.6$ & $38.5 \pm 11.3$ & $39.1 \pm 11.8$ & $37.5 \pm 12.0$ & $40.1 \pm 12.2$ \\
\hline Body weight (kg) & $57.8 \pm 11.4$ & $62.8 \pm 13.5$ & $56.6 \pm 8.1$ & $55.1 \pm 12.7$ & $55.4 \pm 12.1$ & $56.2 \pm 12.1$ & $57.0 \pm 11.5$ \\
\hline Duration of UC (yr) & $3.9(1.6-11.1)$ & $6.5(0.8-10.2)$ & $2.7(0.5-14.1)$ & $2.5(0.7-16.6)$ & $2.8(0.6-8.7)$ & $3.0(0.7-16.6)$ & $3.1(0.5-14.1)$ \\
\hline \multicolumn{8}{|l|}{ Concomitant medications for UC } \\
\hline Only glucocorticoids & $6(32)$ & $3(27)$ & $6(32)$ & $9(31)$ & $11(31)$ & $15(31)$ & $20(31)$ \\
\hline Only immunomodulators & $7(37)$ & $2(18)$ & $5(26)$ & $6(21)$ & $13(37)$ & $13(27)$ & $20(31)$ \\
\hline $\begin{array}{l}\text { Glucocorticoids and } \\
\text { immunomodulators }\end{array}$ & $5(26)$ & $3(27)$ & $3(16)$ & $8(28)$ & $3(9)$ & $13(27)$ & $9(14)$ \\
\hline $\begin{array}{l}\text { No glucocorticoids or } \\
\text { immunomodulators }\end{array}$ & $1(5)$ & $3(27)$ & $5(26)$ & $6(21)$ & $8(23)$ & $7(15)$ & $16(25)$ \\
\hline Patients with prior anti-TNF use & $2(11)$ & 0 & $1(5)$ & $2(7)$ & $3(9)$ & $4(8)$ & $4(6)$ \\
\hline Patients with prior anti-TNF failure & $2(11)$ & 0 & 0 & $2(7)$ & $1(3)$ & $4(8)$ & $1(2)$ \\
\hline Complete Mayo score & $8.3 \pm 1.8$ & $8.1 \pm 1.1$ & $8.5 \pm 1.5$ & $8.3 \pm 1.3$ & $9.1 \pm 1.5$ & $8.3 \pm 1.5$ & $8.8 \pm 1.5$ \\
\hline
\end{tabular}

Values are presented as number (\%), mean \pm standard deviation, or median (range).

Intent-to-treat $(I T T)=$ patients who showed response to vedolizumab at 6 weeks and were randomized as part of the double-blind maintenance phase (maintenance ITT population); Non-ITT placebo = patients that were randomized to placebo during the induction phase and continued to received doubleblind placebo during maintenance phase (maintenance safety population only); Non-IT vedolizumab q4W=patients that did not show response to vedolizumab at 6 weeks and received open-label vedolizumab during the maintenance phase (maintenance safety population only); Placebo combined=all patients that received placebo during the maintenance phase; Vedolizumab combined =all patients that received vedolizumab during the maintenance phase.

${ }^{a}$ Data for the ITT population.

'Patient numbers do not exactly match those shown in disposition (Fig. 1) because those patients who were discontinued from the study during the induction phase continued to be included in the safety population and have been counted within these groups.

q4w, every 4 weeks; q8w, every 8 weeks; UC, ulcerative colitis; TNF, tumor necrosis factor. 
ceived prior treatment with anti-TNF agents.

\section{3) Efficacy}

The efficacy results in the Asian subgroup are shown in Fig. 2. During the induction phase, of the patients in the induction ITT population (Fig. 2A), 55.2\% (95\% CI, 73.3\%-37.1\%) of patients in the vedolizumab group achieved clinical response at week 6 compared to $24.1 \%(95 \% \mathrm{CI}, 8.6 \%-39.7 \%)$ in the placebo group (difference between vedolizumab and placebo $=31.0 \%$; $95 \% \mathrm{CI}$, 7.2\%-54.9\%). The proportions were numerically higher with vedolizumab than placebo for patients achieving clinical remission (24.1\% vs. $3.4 \%)$ and mucosal healing (51.7\% vs. $31.0 \%)$.

In the maintenance phase, the clinical remission and mucosal healing rates at 52 weeks in the vedolizumab q8w group were $9.1 \%$ (95\% CI, $0.2 \%-41.3 \%$ ) and $45.5 \%$ (95\% CI, 16.7\%$76.6 \%$ ), respectively, in the maintenance ITT population (Fig. $2 B)$. None of the 11 patients in the vedolizumab q8w group attained durable clinical remission. Efficacy rates were generally numerically higher in the vedolizumab q4w group compared to placebo. In patients in the vedolizumab q4w group, the clinical remission and mucosal healing rates were $36.8 \%$ (95\% CI, $15.2 \%-58.5 \%$ ) and $47.4 \%$ (95\% CI, 24.9\%-69.8\%), respectively; the corresponding rates in the placebo group were $31.6 \%$ (95\% CI, 10.7\%-52.5\%) and 47.4\% (95\% CI, 24.9\%-69.8\%), respectively. Durable clinical response was achieved by over $50 \%$ of patients in both vedolizumab groups $(54.5 \%$ in the ve- dolizumab q8w group and $52.6 \%$ in the vedolizumab q4w group) compared to $36.8 \%$ in the placebo group.

\section{4) Safety}

Results regarding the frequency of AEs in each group of the safety population within the Asian subgroup are available in Supplementary Tables 1 and 2. During induction, 35.7\% of patients who received vedolizumab (vedolizumab cohorts 1 and 2 combined) and $34.5 \%$ of patients who received placebo experienced an AE. During maintenance, AEs were reported in $76.9 \%$ of all vedolizumab-treated patients compared to $79.2 \%$ of all patients who received placebo during this period. The frequency of serious infections with vedolizumab was low during the study, with no events experienced in the induction phase, and $3.1 \%$ in the maintenance phase; the corresponding frequencies in the placebo group were $0 \%$ and $6.3 \%$, respectively. During the induction phase, AEs affecting at least $5 \%$ of patients receiving vedolizumab in the safety population were (vedolizumab vs. placebo)-nasopharyngitis (6.0\% vs. 0\%), anemia $(6.0 \%$ vs. $3.4 \%)$ and headache $(6.0 \%$ vs. $0 \%)$. During the maintenance phase, AEs affecting at least $10 \%$ of patients receiving vedolizumab in the safety population were (vedolizumab vs. placebo)-nasopharyngitis ( $12.3 \%$ vs. $4.2 \%$ ) and upper respiratory tract infection (12.3\% vs. 8.4\%).
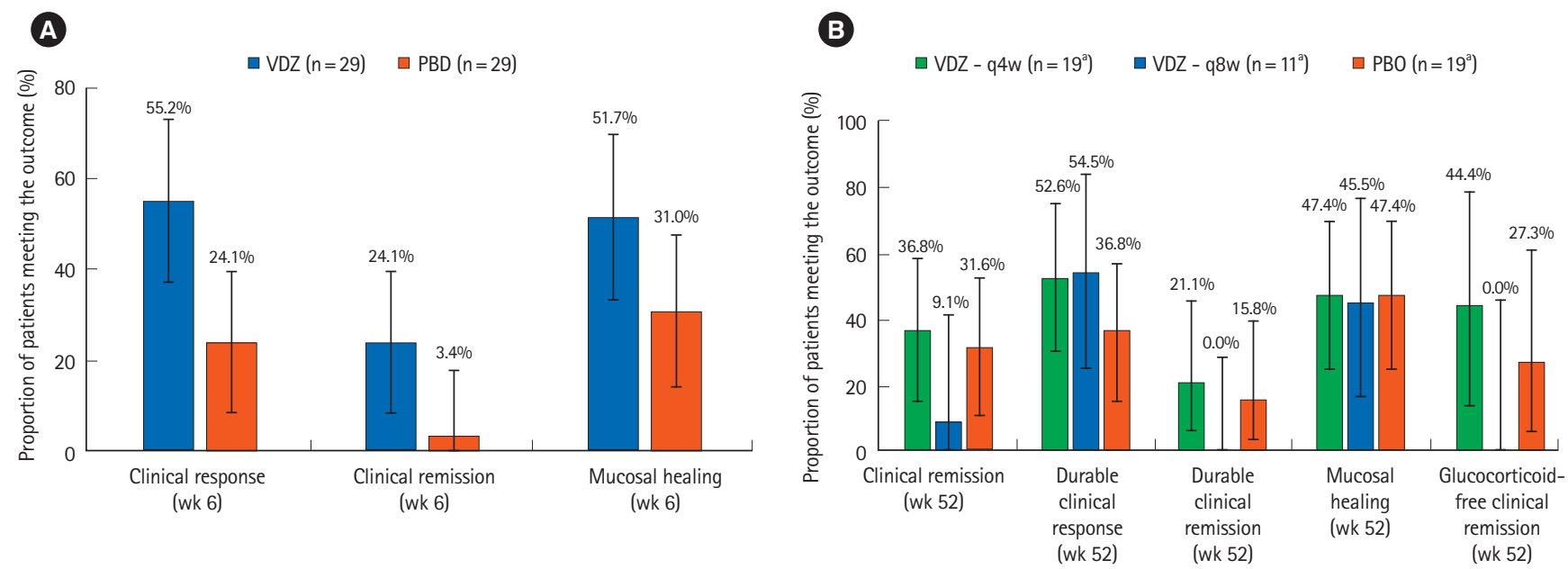

Fig. 2. Comparison of efficacy results for vedolizumab versus placebo in the Asian countries subgroup in GEMINI 1 patients (A) in the induction phase, in the induction intent-to-treat (ITT) population, the rates of clinical response, clinical remission and mucosal healing were numerically higher with vedolizumab compared to placebo, (B) in the maintenance phase, in the maintenance IT population, the efficacy rates were numerically higher with vedolizumab q4w compared to placebo. Error bars represent $95 \%$ confidence intervals. ${ }^{\mathrm{a} F o r}$ glucocorticoid-free remission, the analysis was restricted to patients who were on glucocorticoids at baseline; therefore the " $n$ " numbers for VDZ $q 4 w_{1}$ VDZ q8w, and PBO were 9, 6, and 11, respectively. VDZ, vedolizumab; PBO, placebo; $q 4 w_{1}$ every 4 weeks; q8w, every 8 weeks. 


\section{Non-Asian Countries Subgroup 1) Disposition}

The disposition of the non-Asian subgroup of the GEMINI 1 study is shown in Fig. 1. Cohort 1 consisted of 316 patients, of whom 196 were randomized to vedolizumab and 120 to placebo (induction ITT population). Cohort 2 consisted of an additional 466 patients who received treatment with open-label vedolizumab; they were included only in the safety population for induction. Three hundred and twenty-four patients (48.9\%) showed response to vedolizumab at 6 weeks and were randomized in the maintenance phase- 106 to vedolizumab q4w, 111 to vedolizumab 8qw and 107 to placebo (maintenance ITT population). Three hundred patients (45.3\%) failed to show response to vedolizumab at 6 weeks and received openlabel vedolizumab during maintenance; 38 patients (5.7\%) who were treated with vedolizumab discontinued the study during the induction phase. One hundred and ten patients who received placebo during induction continued to receive it during maintenance.

\section{2) Demography and Baseline Characteristics}

As seen in Tables 3 and 4, the median duration of UC was 5.0 years (range, 0.5-25.8 years) in the vedolizumab cohort 1 and 5.2 years (range, $0.5-38.5$ years) in the placebo group. Prior treatment with glucocorticoids and/or immunomodulators had been received by $67.9 \%$ and $65.8 \%$ of the patients in the vedolizumab cohort 1 and placebo groups, respectively; while prior treatment with anti-TNF agents had been received by $48.5 \%$ and $59.2 \%$ patients, respectively.

\section{3) Efficacy}

Fig. 3 shows the efficacy results in the non-Asian subgroup. In the induction phase (Fig. 3A), the clinical response rates in the vedolizumab cohort 1 and placebo group were $45.9 \%$ (95\% CI, 38.9\%-52.9\%) and 25.8\% (95\% CI, 18.0\%-33.7\%), respectively (difference between vedolizumab and placebo $=20.1 \%$; $95 \%$ CI, 9.6\%-30.6\%). Similar results were observed with other efficacy outcomes-clinical remission rates were $15.8 \%$ in vedolizumab versus $5.8 \%$ in placebo; and mucosal healing rates were $39.3 \%$ in vedolizumab versus $23.3 \%$ in placebo.

In the maintenance phase (Fig. 3B), the efficacy rates were higher in both vedolizumab groups compared to placebo for all outcomes-as examples, clinical remission rates in vedolizumab q4w, vedolizumab q8w and placebo groups were $46.2 \%, 45.0 \%$, and $13.1 \%$, respectively; and mucosal healing rates were $57.5 \%, 52.3 \%$, and $15.0 \%$, respectively.

\section{4) Safety}

$\mathrm{AE}$ frequencies for the safety population within the non-Asian subgroup are presented in Supplementary Tables 3 and 4. During induction, AEs were reported by $46.4 \%$ of those who received vedolizumab and $49.2 \%$ of those who received placebo; the corresponding frequencies during maintenance were $80.5 \%$ and $80.2 \%$, respectively. Serious infections with vedolizumab were reported by $0.6 \%$ during induction, and by $1.8 \%$ during maintenance; with placebo, the corresponding frequencies were $2.5 \%$ and $1.9 \%$, respectively. During the induction phase, headache (7.9\% in vedolizumab, $5.8 \%$ in placebo) was the only $\mathrm{AE}$ affecting at least $5 \%$ of patients receiving vedolizumab in the safety population. During the maintenance phase, AEs affecting at least $10 \%$ of patients receiving vedolizumab in the safety population were nasopharyngitis ( $13.0 \%$ in vedolizumab, $10.6 \%$ in placebo), exacerbation of UC (16.9\%, 22.5\%), and headache (13.7\%, 11.0\%).

\section{Comparison of Asian and Non-Asian Countries Subgroups \\ 1) Demography and Baseline Characteristics}

The body weight was lower and duration of UC shorter in the Asian subgroup compared to the non-Asian subgroup (Tables 1-4). The patients in the Asian subgroup were slightly younger than those in the non-Asian subgroup. Importantly, prior antiTNF use was much more frequent in the non-Asian compared to Asian subgroup. Disease severity (based on complete Mayo score) was broadly comparable across the 2 subgroups.

\section{2) Efficacy}

The efficacy comparisons between the Asian and non-Asian subgroups are shown in Fig. 4. In the induction phase (Fig. $4 \mathrm{~A}$ ), the rates of clinical response, clinical remission and mucosal healing were numerically higher in Asian subgroup compared to non-Asian-the rates for vedolizumab-treated patients in the Asian subgroup versus non-Asian subgroup were $55.2 \%$ (95\% CI, 37.1\%-73.3\%) versus $45.9 \%$ (95\% CI, 38.9\%-52.9\%) for clinical response, $24.1 \%$ (95\% CI, 8.6\%-39.7\%) versus $15.8 \%$ (95\% CI, 10.7\%-20.9\%) for clinical remission and 51.7\% (95\% CI, 33.5\%-69.9\%) versus 39.3\% (95\% CI, 32.4\%-46.1\%) for mucosal healing.

In the maintenance phase, no clear trend was seen for comparisons between the 2 subgroups. Fig. $4 \mathrm{~B}$ shows the comparison between the vedolizumab arms (q8w, q4w) in the Asian versus non-Asian subgroups. Across all patients, the efficacy rates were comparably high for durable clinical response 
Table 3. Characteristics in the Non-Asian Countries Subgroup of GEMINI 1 Patients: Induction Phase

\begin{tabular}{lcccc}
\hline Parameter & Placebo $^{\mathrm{a}}$ & $\begin{array}{c}\text { Vedolizumab } \\
\text { (cohort 1) }\end{array}$ & $\begin{array}{c}\text { Vedolizumab } \\
\text { (cohort 2) }\end{array}$ & $\begin{array}{c}\text { Vedolizumab } \\
\text { (combined) }\end{array}$ \\
\hline No. & 120 & 196 & 466 & 662 \\
Male sex & $79(66)$ & $115(59)$ & $272(58)$ & $387(58)$ \\
Age (yr) & $41.8 \pm 12.7$ & $40.7 \pm 13.3$ & $40.1 \pm 13.3$ & $40.2 \pm 13.3$ \\
Body weight (kg) & $76.6 \pm 16.1$ & $74.6 \pm 16.7$ & $76.2 \pm 19.0$ & $75.7 \pm 18.4$ \\
Duration of UC (yr) & $5.2(0.5-38.5)$ & $5.0(0.5-25.8)$ & $5.4(0.5-37.5)$ & $5.2(0.5-37.5)$ \\
Concomitant medications for UC & & & & \\
$\quad$ Only glucocorticoids & $49(41)$ & $73(37)$ & $175(38)$ & $248(37)$ \\
$\quad$ Only immunomodulators & $12(10)$ & $20(10)$ & $94(20)$ & $114(17)$ \\
$\quad$ Glucocorticoids and immunomodulators & $18(15)$ & $40(20)$ & $69(15)$ & $109(16)$ \\
$\quad$ No glucocorticoids or immunomodulators & $41(34)$ & $63(32)$ & $128(27)$ & $191(29)$ \\
Patients with prior anti-TNF use & $71(59)$ & $95(48)$ & $257(55)$ & $352(53)$ \\
Patients with prior anti-TNF failure & $61(51)$ & $82(42)$ & $219(47)$ & $352(53)$ \\
Complete Mayo score & $8.7 \pm 1.8$ & $8.5 \pm 1.8$ & $8.6 \pm 1.8$ & $8.5 \pm 1.8$ \\
\hline
\end{tabular}

Values are presented as number (\%), mean \pm standard deviation, or median (range).

Placebo and vedolizumab (cohort 1)=the groups that were part of the double-blind induction phase (induction intent-to-treat [IT] population); Vedolizumab (cohort 2)=additional patients were enrolled to meet the maintenance phase sample size requirements and received open-label vedolizumab (induction safety population only); Vedolizumab combined = all patients that received vedolizumab during the induction phase.

${ }^{a}$ Data for the ITT population.

UC, ulcerative colitis; TNF, tumor necrosis factor.

Table 4. Characteristics in the Non-Asian Countries Subgroup of GEMINI 1 Patients: Maintenance Phase

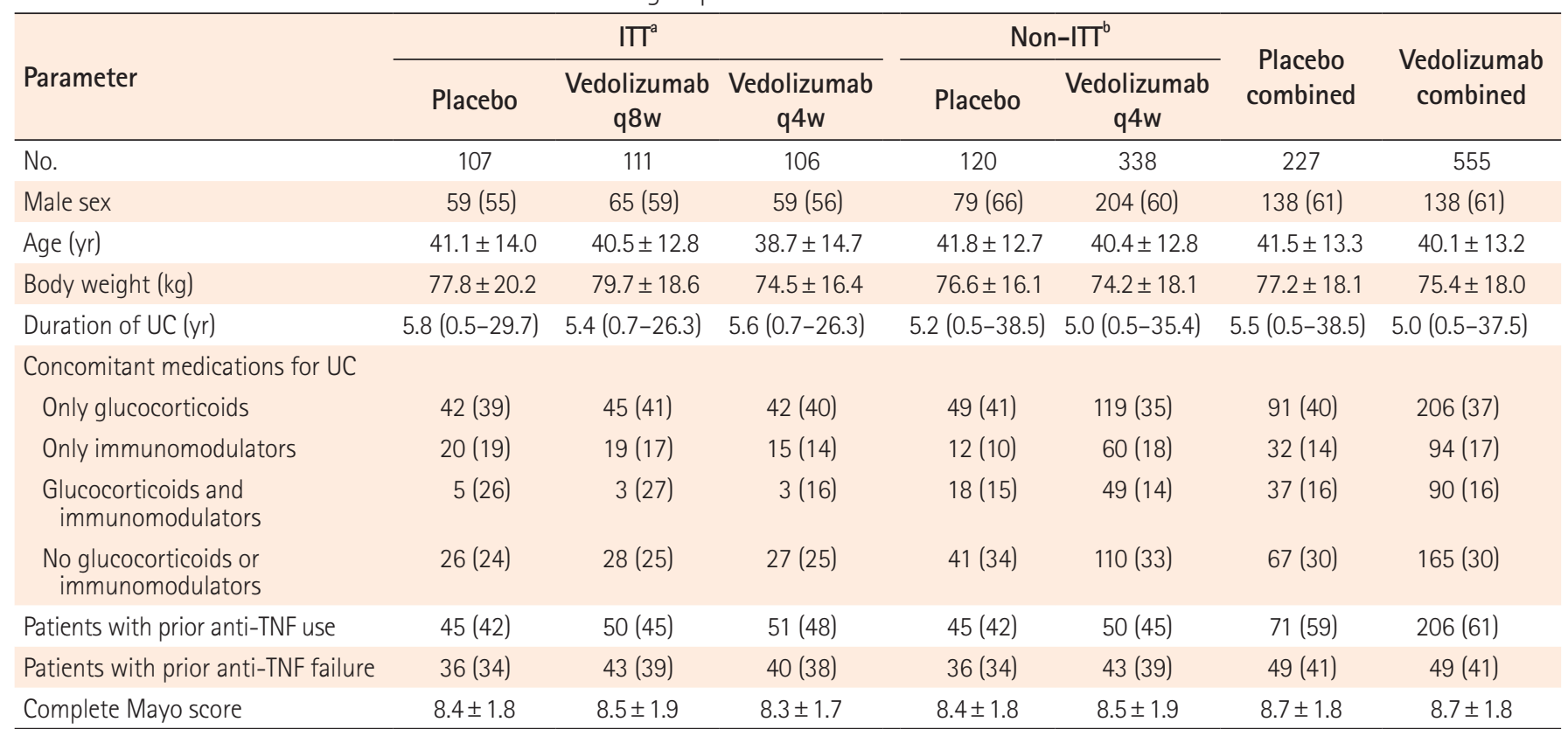

Values are presented as number (\%), mean \pm standard deviation, or median (range).

Intent-to-treat $(I T)=$ patients who showed response to vedolizumab at 6 weeks and were randomized as part of the double-blind maintenance phase (maintenance ITT population); Non-ITT placebo= patients that were randomized to placebo during the induction phase and continued to received double-blind placebo during maintenance phase (maintenance safety population only); Non-ITT vedolizumab q4w=patients that did not show response to vedolizumab at 6 weeks and received open-label vedolizumab during the maintenance phase (maintenance safety population only); Placebo combined = all patients that received placebo during the maintenance phase; Vedolizumab combined = all patients that received vedolizumab during the maintenance phase.

a'Data for the ITT population.

${ }^{b}$ Patient numbers do not exactly match those shown in disposition (Fig. 1) because those patients who were discontinued from the study during the induction phase continued to be included in the safety population and have been counted within these groups.

q4w, every 4 weeks; $98 \mathrm{w}$, every 8 weeks; UC, ulcerative colitis; TNF, tumor necrosis factor. 

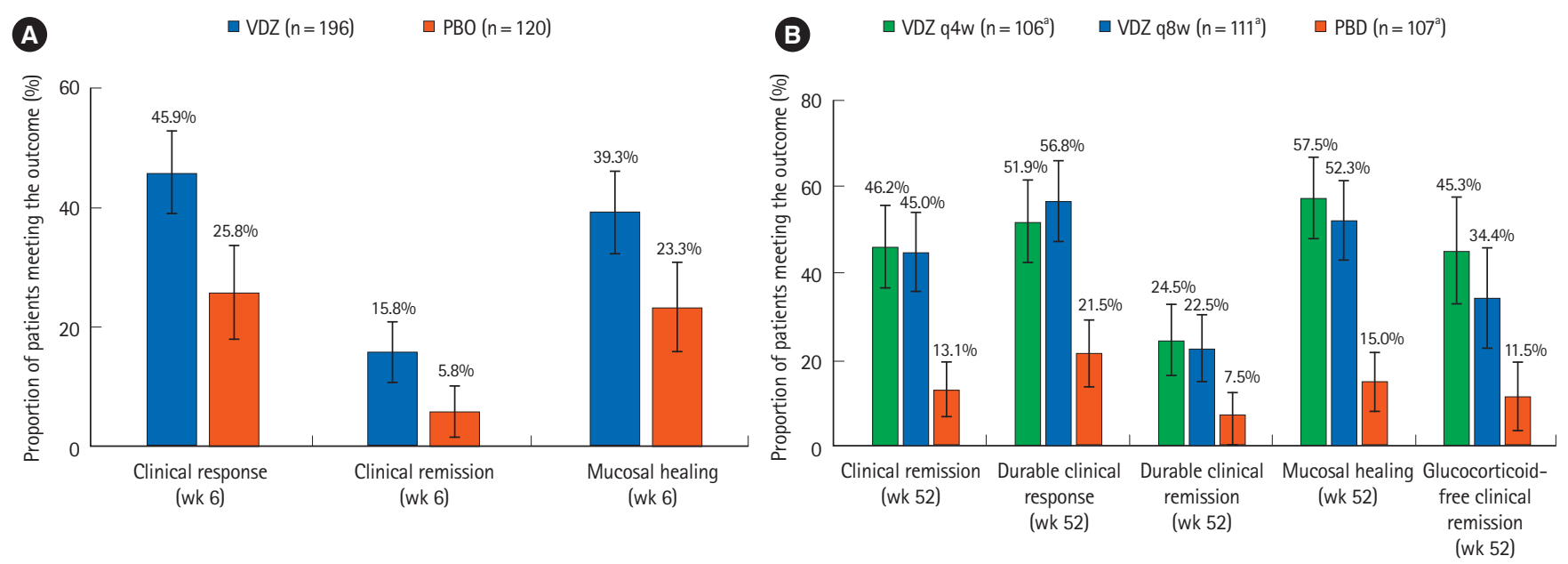

Fig. 3. Comparison of efficacy results for vedolizumab versus placebo in the non-Asian countries subgroup in GEMINI 1 patients (A) in the induction phase, in the induction intent-to-treat (ITT) population, the rates of clinical response, clinical remission and mucosal healing were numerically higher with vedolizumab compared to placebo, (B) in the maintenance phase, in the maintenance IT population, the efficacy rates were higher in both vedolizumab groups compared to placebo for all outcomes. Error bars represent 95\% confidence

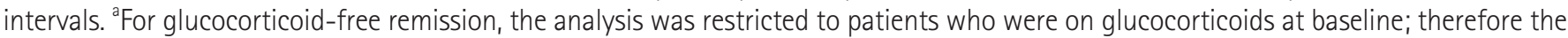
" $n$ " numbers for VDZ q4w, VDZ q8w and PBO were 64, 64 and 61, respectively. VDZ, vedolizumab; PBO, placebo; q4w, every 4 weeks; q8w, every 8 weeks.
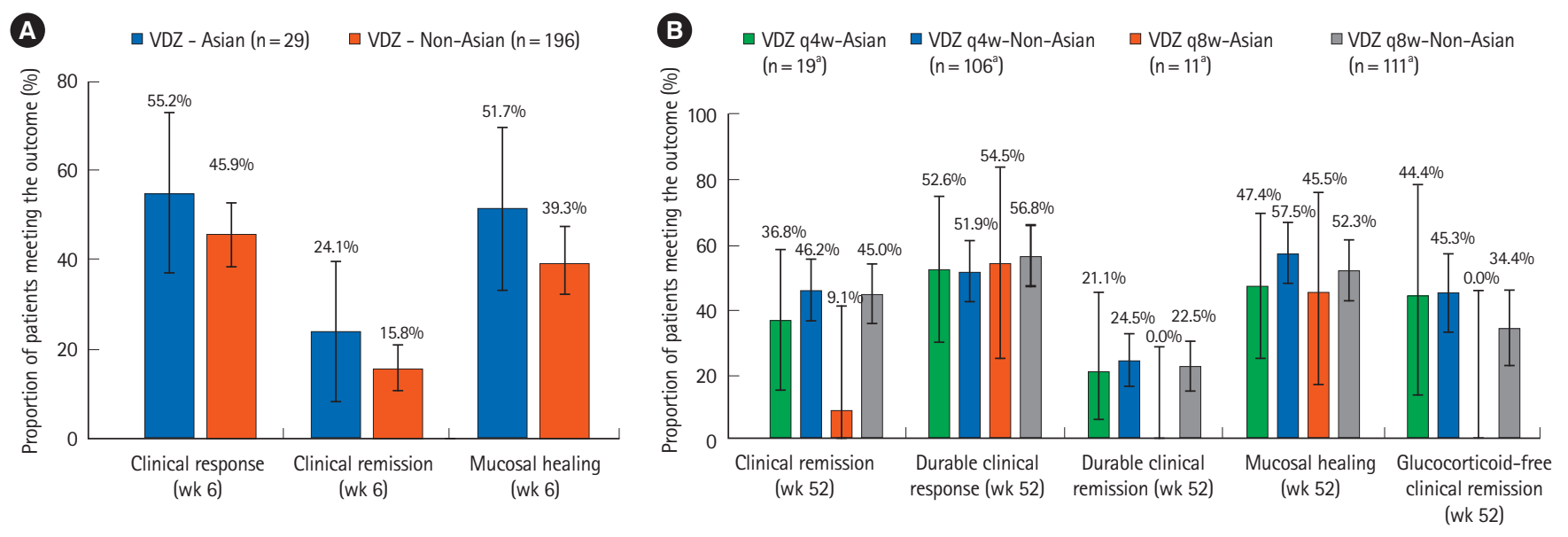

Fig. 4. Comparison of efficacy results in the vedolizumab arms of Asian versus non-Asian countries subgroups in GEMINI 1 patients (A) in the induction phase, the rates of clinical response, clinical remission and mucosal healing were numerically higher in the Asian compared to non-Asian subgroup, (B) in the maintenance phase, no clear trend was visible for comparisons between the 2 subgroups. Error bars represent 95\% confidence intervals. aFor glucocorticoid-free remission, the analysis was restricted to patients who were on glucocorticoids at baseline; therefore the " $n$ " numbers for VDZ q4w-Asian, VDZ q4w-non-Asian, VDZ q8w-Asian, and VDZ q8w-non-Asian were $9,64,6$, and 64, respectively. VDZ, vedolizumab; PBO, placebo; q4w, every 4 weeks; q8w, every 8 weeks.

(51.9\% to $56.8 \%)$ and mucosal healing (45.5\% to $57.5 \%)$, and relatively low for durable clinical remission ( $0 \%$ to $24.5 \%)$.

\section{3) Safety}

A comparison of the frequency of AEs in the Asian and nonAsian subgroups (Supplementary Tables 1-4) showed that the AE frequency during induction was numerically lower in Asian compared to non-Asian subgroups in the vedolizumab groups (35.7\% vs. $46.4 \%$, respectively) and in the placebo groups (34.5\% vs. $49.2 \%$, respectively). In the induction phase, among those treated with vedolizumab, serious adverse events (SAEs) were reported by $1.2 \%$ of those in the Asian subgroup and $3.6 \%$ of those in the non-Asian subgroup; the corresponding frequencies during the maintenance phase were $13.8 \%$ and $12.3 \%$, re- 
spectively. The frequencies of serious infections in vedolizumab-treated patients in the Asian versus non-Asian subgroups were $0 \%$ versus $0.6 \%$ in the induction phase, and $3.1 \%$ versus $1.8 \%$ in the maintenance phase. Headache was a common $\mathrm{AE}$ reported in vedolizumab-treated patients in both Asian and non-Asian subgroups in the induction phase (6.0\% vs. $7.9 \%$, respectively).

\section{DISCUSSION}

This post-hoc exploratory analysis assessed the outcomes in patients from 6 Asian countries that had participated in the GEMINI 1 study.

During induction treatment in the Asian subgroup, efficacy rates with vedolizumab were numerically higher than with placebo on the primary outcome of clinical response at 6 weeks, as well as on the other outcomes-clinical remission and mucosal healing. For all 3 induction outcomes, the efficacy rates with vedolizumab were also numerically higher in the Asian subgroup compared to the non-Asian subgroup-with a difference of $9.3 \%, 8.3 \%$, and $12.4 \%$, respectively, for clinical response, clinical remission and mucosal healing. In this context, it is important to note that the Asian subgroup patients were slightly younger, and had experienced UC for fewer years than their non-Asian counterparts. Perhaps more importantly, the prior use of anti-TNF agents differed sharply across the 2 groups, with a lower frequency of prior anti-TNF use (and failure) in the Asian compared to non-Asian subgroups. Prior anti-TNF use and failure may suggest a worsening, potentially refractory disease, and a previous post-hoc analysis of GEMINI 1 data demonstrated that vedolizumab had higher efficacy in TNF-naïve patients compared to those with prior anti-TNF use. ${ }^{16}$ Therefore, the slightly higher efficacy rates observed with vedolizumab in the Asian subgroup could in part be explained by a greater TNF-naïve sample.

In terms of the efficacy with vedolizumab in the Asian subgroup in the maintenance phase, drawing any definitive conclusions from this analysis is not possible due to the small sample sizes involved, i.e., only 11 patients provided maintenance data for vedolizumab administered $\mathrm{q} 8 \mathrm{w}$ in the Asian subgroup. The efficacy rates on all outcomes were comparable to or numerically slightly higher than placebo for patients randomized to 4-weekly vedolizumab. For patients randomized to 8-weekly vedolizumab, the results were inconsistent-the durable clinical response rate was higher than placebo and mucosal healing rate was comparable; however, the clinical remission, durable clinical remission and glucocorticoid-free remission rates were lower than placebo, with wide overlapping CIs. Notably, the median disease duration was higher in patients randomized to 8-weekly vedolizumab (6.5 years) compared to those randomized to placebo (3.9 years) or 4-weekly vedolizumab (2.7 years), possibly indicating that the disease process was further advanced in the patients receiving 8-weekly vedolizumab. Persistence of symptoms on patient-reported outcomes despite mucosal healing has been reported in UC. ${ }^{17}$ Patients with more advanced disease are more likely to experience complications such as anorectal and motility dysfunction due to structural and functional damage beyond the mucosal layer, and can therefore experience symptoms despite mucosal healing. Earlier and more aggressive treatment of the disease may be needed to prevent these complications. ${ }^{18}$

In terms of comparisons between Asian and non-Asian patients in the maintenance phase, although no consistent trends were seen overall, in patients receiving 8-weekly vedolizumab, the rates of clinical remission, durable clinical remission and glucocorticoid-free clinical remission were lower in the Asian subgroup compared to the non-Asian subgroup. Given the overlapping 95\% CIs for these comparisons and the small sample size in the Asian 8-weekly vedolizumab group, it is difficult to derive any meaningful conclusions from these data. It is pertinent to note, however, that in patients randomized to 8-weekly vedolizumab, the median disease duration was slightly higher in the Asian subgroup (6.5 years) than the non-Asian subgroup (5.4 years); in contrast, the disease duration was lower for the Asian subgroup compared to the non-Asian subgroup, in patients randomized to placebo (3.9 vs. 5.8 years, respectively) and 4-weekly vedolizumab (2.7 vs. 5.6 years, respectively).

The safety data for the Asian subgroup were consistent with the established safety profile of vedolizumab. The rate of AEs and SAEs was comparable in vedolizumab and placebo arms, both in the induction and the maintenance phase. Importantly, the rate of serious infections with vedolizumab was low in the study, and numerically lower than in the placebo arms. The AEs commonly reported were similar across Asian and non-Asian subgroups, and both subgroups showed comparable rates of SAEs and serious infections.

The clinical response rate with vedolizumab in the Asian subgroup in our study (55.2\%) is higher than that reported in a recent study from Japan where they assessed clinical response at 10 weeks after treatment initiation with vedolizumab in patients with UC, and reported a rate of $39.6 \% .{ }^{19}$ This study in- 
cluded a larger sample of patients compared to our study (292 patients enrolled in the induction phase) and they found a statistically significant benefit of vedolizumab over placebo in the maintenance phase. Prior anti-TNF agent use in this study was approximately $50 \%$, which was much higher than in the Asian subgroup in our study (7.1\%); this may potentially explain some of the difference in the clinical response rates between this study and the Asian subgroup in our analysis. In this study in Japan, the clinical response rate with vedolizumab at 10 weeks was $53.2 \%$ in those without prior anti-TNF use and $27.1 \%$ in those with prior anti-TNF use.

A recent study from Singapore has reported real-world data on the use of vedolizumab in patients with $\mathrm{IBD}^{20}$ In this study, in patients with UC $(\mathrm{n}=25)$, steroid-free complete remission was achieved by $68.0 \%, 66.7 \%$, and $80.0 \%$ of patients after 14 , 24 , and 54 weeks, respectively, of vedolizumab treatment. Treatment with vedolizumab was well-tolerated. This study provides some real-world data on the effectiveness and safety of vedolizumab in UC patients which are consistent with that seen in patients from Asian countries in our study. Another real-world study, conducted in South Korea, focused on treatment-refractory IBD patients (UC, $\mathrm{n}=18$ ), all of whom had failed prior anti-TNF therapy; ${ }^{21}$ over 57 weeks of treatment, induction and maintenance therapy with vedolizumab appeared to be effective, with an acceptable safety profile. Given the relative paucity of literature, additional real-world evidence on the use of vedolizumab for UC in Asian countries are still needed.

The key limitation of our analysis is the small sample size of the Asian subgroup, which was reflected in the wide CIs for this subgroup. The small sample size precluded any formal statistical comparison of the vedolizumab-placebo differences across the Asian and non-Asian subgroups, and particularly impacted the robustness of the safety comparisons. While this limits the ability to draw definitive conclusions from the data, trends can be observed which can drive further research in this area.

In summary, in patients from Asian countries, the efficacy and safety of vedolizumab in treatment of UC were broadly consistent with that in the overall study population in the GEMINI 1 study.

\section{ADDITIONAL INFORMATION}

\section{Funding Source}

The study was funded by Takeda Pharmaceuticals Company Ltd.

\section{Conflict of Interest}

Clinical trials were sponsored and conducted by Takeda Pharmaceuticals Company Ltd. Medical writing support was sponsored by Takeda Pharmaceuticals Company Ltd. Ooi CJ has received speaker and travel grants from Takeda, Johnson and Johnson, Celltrion, Abbvie, Shire, and Ferring Pharmaceuticals; and participated in Advisory Boards for Takeda, Johnson and Johnson, Shire, and Ferring Pharmaceuticals. Dirk Demuth, Dirk Lindner, and Shashi Adsul are employees of Takeda Pharmaceuticals Company Ltd. The other authors report no conflict of interest.

Ooi $\mathrm{CJ}$ is an editorial board member of the journal but did not involve in the peer reviewer selection, evaluation, or decision process of this article. No other potential conflicts of interest relevant to this article were reported.

\section{Author Contribution}

Conceptualization: Ooi CJ, Hilmi IN, Kim HJ, Jalihal U, Wu DC, Demuth D, Lindner D, Adsul S. Methodology: Ooi CJ, Hilmi IN, Kim HJ, Jalihal U, Wu DC, Demuth D, Lindner D, Adsul S. Formal analysis: Demuth D, Lindner D, Adsul S. Project administration: Ooi CJ, Demuth D, Lindner D, Adsul S. Visualization: Ooi CJ, Hilmi IN, Kim HJ, Jalihal U, Wu DC, Demuth D, Lindner D, Adsul S. Writing - original draft: Demuth D, Lindner D, Adsul S. Writing - review and editing: Ooi CJ, Hilmi IN, Kim HJ, Jalihal U, Wu DC, Demuth D, Lindner D, Adsul S. Approval of final manuscript: all authors.

\section{Others}

The authors thank the patients and their caregivers in addition to the investigators and their teams who contributed to this study. Medical writing support was provided by Assansa, India (a Healthcare Consultancy-Assansa consultants Dr. Aamir Shaikh MD, Dr. Saifuddin Kharawala MBBS, DPM) and sponsored by Takeda Pharmaceuticals Company Ltd.

\section{ORCID}

Ooi CJ

Hilmi IN

Kim HJ

Jalihal U

Wu DC

Demuth D

Lindner D

Adsul S https://orcid.org/0000-0001-8961-5455 https://orcid.org/0000-0001-7091-0032 https://orcid.org/0000-0002-9675-4557 https://orcid.org/0000-0002-5983-4750 https://orcid.org/0000-0003-3742-0634 https://orcid.org/0000-0002-2415-2135 https://orcid.org/0000-0003-2593-1322 https://orcid.org/0000-0002-0593-1088 


\section{Supplementary Material}

Supplementary materials are available at the Intestinal Research website (https://www.irjournal.org).

\section{REFERENCES}

1. National Institute for Health and Care Excellence. Ulcerative colitis: management (NICE Guideline CG166) [Internet]. c2013 [cited 2018 Apr 10]. https: //www.nice.org.uk/guidance/cg166.

2. Feuerstein JD, Cheifetz AS. Ulcerative colitis: epidemiology, diagnosis, and management. Mayo Clin Proc 2014;89:15531563.

3. Prideaux L, Kamm MA, De Cruz PP, Chan FK, Ng SC. Inflammatory bowel disease in Asia: a systematic review. J Gastroenterol Hepatol 2012;27:1266-1280.

4. Ng SC, Kaplan GG, Tang W, et al. Population density and risk of inflammatory bowel disease: a prospective populationbased study in 13 countries or regions in Asia-Pacific. Am J Gastroenterol 2019;114:107-115.

5. Yang SK, Yun S, Kim JH, et al. Epidemiology of inflammatory bowel disease in the Songpa-Kangdong district, Seoul, Korea, 1986-2005: a KASID study. Inflamm Bowel Dis 2008;14:542549.

6. Wong U, Cross RK. Primary and secondary nonresponse to infliximab: mechanisms and countermeasures. Expert Opin Drug Metab Toxicol 2017;13:1039-1046.

7. Ford AC, Peyrin-Biroulet L. Opportunistic infections with anti-tumor necrosis factor- $\alpha$ therapy in inflammatory bowel disease: meta-analysis of randomized controlled trials. Am J Gastroenterol 2013;108:1268-1276.

8. World Health Organization. Global tuberculosis report 2017 [Internet]. c2017 [cited 2018 Apr 10]. http://www.who.int/tb/ publications/global_report/en/.

9. Ungar B, Kopylov U. Advances in the development of new biologics in inflammatory bowel disease. Ann Gastroenterol 2016;29:243-248.

10. Feagan BG, Rutgeerts $P$, Sands BE, et al. Vedolizumab as induction and maintenance therapy for ulcerative colitis. N Engl J Med 2013;369:699-710.

11. Schreiber S, Dignass A, Peyrin-Biroulet L, et al. Systematic re- view with meta-analysis: real-world effectiveness and safety of vedolizumab in patients with inflammatory bowel disease. J Gastroenterol 2018;53:1048-1064.

12. Ng SC, Hilmi IN, Blake A, et al. Low frequency of opportunistic infections in patients receiving vedolizumab in clinical trials and post-marketing setting. Inflamm Bowel Dis 2018;24: 2431-2441.

13. Cheon JH. Understanding the complications of anti-tumor necrosis factor therapy in East Asian patients with inflammatory bowel disease. J Gastroenterol Hepatol 2017;32:769-777.

14. Leung WK, Ng SC, Chow DK, et al. Use of biologics for inflammatory bowel disease in Hong Kong: consensus statement. Hong Kong Med J 2013;19:61-68.

15. Leung WK. Optimization of inflammatory bowel disease cohort studies in Asia. Intest Res 2015;13:208-212.

16. Feagan BG, Rubin DT, Danese S, et al. Efficacy of vedolizumab induction and maintenance therapy in patients with ulcerative colitis, regardless of prior exposure to tumor necrosis factor antagonists. Clin Gastroenterol Hepatol 2017;15:229239.

17. Colombel JF, Keir ME, Scherl A, et al. Discrepancies between patient-reported outcomes, and endoscopic and histological appearance in UC. Gut 2017;66:2063-2068.

18. Torres J, Billioud V, Sachar DB, Peyrin-Biroulet L, Colombel JF. Ulcerative colitis as a progressive disease: the forgotten evidence. Inflamm Bowel Dis 2012;18:1356-1363.

19. Motoya S, Watanabe K, Ogata H, et al. Vedolizumab in Japanese patients with ulcerative colitis: a phase 3 , randomized, double-blind, placebo-controlled study. PLoS One 2019;14: e0212989.

20. Gan AT, Chan WP, Ling KL, et al. P634 Real-world data on the efficacy and safety of vedolizumab therapy in patients with inflammatory bowel disease: a retrospective nation-wide cohort study in Singapore. J Crohns Colitis 2019;13(Suppl 1): S434-S435.

21. Kim J, Ham NS, Oh EH, et al. P277 Real life effectiveness and safety of vedolizumab induction and maintenance therapy for Korean IBD patients in whom anti-TNF treatment failed: a prospective cohort study. J Crohns Colitis 2019;13(Suppl 1): S237. 


\section{See "Efficacy and safety of vedolizumab in ulcerative colitis in patients from Asian countries in the GEMINI 1 study" on page 71-82.}

Supplementary Table 1. Key Safety Results in the Asian Countries Subgroup of GEMINI 1 Patients: Induction Phase

\begin{tabular}{|c|c|c|c|c|}
\hline Parameter & Placebo $^{a}$ & $\begin{array}{c}\text { Vedolizumab } \\
\text { (cohort 1) }^{\mathrm{a}}\end{array}$ & $\begin{array}{c}\text { Vedolizumab } \\
\text { (cohort 2) }\end{array}$ & $\begin{array}{c}\text { Vedolizumab } \\
\text { (combined) }\end{array}$ \\
\hline No. & 29 & 29 & 55 & 84 \\
\hline Any AE & $10(34)$ & $6(21)$ & $24(44)$ & $30(36)$ \\
\hline Drug-related AE & $4(14)$ & $3(10)$ & $6(11)$ & $9(11)$ \\
\hline AE resulting in study discontinuation & $1(3)$ & 0 & 0 & 0 \\
\hline SAE & $3(10)$ & 0 & $1(2)$ & $1(1)$ \\
\hline Serious infection AE & 0 & 0 & 0 & 0 \\
\hline Drug-related SAE & $1(3)$ & 0 & 0 & 0 \\
\hline Serious AE resulting in study discontinuation & $1(3)$ & 0 & 0 & 0 \\
\hline Deaths & 0 & 0 & 0 & 0 \\
\hline
\end{tabular}

Values are presented as number (\%).

Placebo and vedolizumab (cohort 1)=the groups that were part of the double-blind induction phase (induction intent-to-treat [ITT] population); Vedolizumab (cohort 2)=additional patients were enrolled to meet the maintenance phase sample size requirements and received open-label vedolizumab (induction safety population only); Vedolizumab combined = all patients that received vedolizumab during the induction phase.

${ }^{a}$ Data for the ITT population.

$A E$, adverse event; $S A E$, serious $A E$. 
Supplementary Table 2. Key Safety Results in the Asian Countries Subgroup of GEMINI 1 Patients: Maintenance Phase

\begin{tabular}{|c|c|c|c|c|c|c|c|}
\hline \multirow[b]{2}{*}{ Parameter } & \multicolumn{3}{|c|}{$\mathrm{IT}^{\mathrm{a}}$} & \multicolumn{2}{|c|}{ Non-ITT' } & \multirow{2}{*}{$\begin{array}{l}\text { Placebo } \\
\text { combined }\end{array}$} & \multirow{2}{*}{$\begin{array}{l}\text { Vedolizumab } \\
\text { combined }\end{array}$} \\
\hline & Placebo & $\begin{array}{l}\text { Vedolizumab } \\
\text { q8w }\end{array}$ & $\begin{array}{l}\text { Vedolizumab } \\
\text { q4w }\end{array}$ & Placebo & $\begin{array}{c}\text { Vedolizumab } \\
\text { q4w }\end{array}$ & & \\
\hline No. & 19 & 11 & 19 & 29 & 35 & 48 & 65 \\
\hline Any AE & $14(74)$ & $8(73)$ & $17(89)$ & $24(83)$ & $25(71)$ & $38(79)$ & $50(77)$ \\
\hline Drug-related AE & $2(11)$ & $2(18)$ & $5(26)$ & $6(21)$ & $7(20)$ & $8(17)$ & $14(22)$ \\
\hline $\begin{array}{l}\text { AE resulting in study } \\
\text { discontinuation }\end{array}$ & 0 & $1(9)$ & $2(11)$ & $2(7)$ & $1(3)$ & $2(4)$ & $4(6)$ \\
\hline SAE & $2(11)$ & $3(27)$ & $2(11)$ & $5(17)$ & $4(11)$ & $7(15)$ & $9(14)$ \\
\hline Serious infection $A E$ & $2(11)$ & $1(9)$ & 0 & $1(3)$ & $1(3)$ & $3(6)$ & $2(3)$ \\
\hline Drug-related SAE & 0 & $1(9)$ & 0 & $1(3)$ & $1(3)$ & $1(2)$ & $2(3)$ \\
\hline $\begin{array}{l}\text { Serious AE resulting in } \\
\text { study discontinuation }\end{array}$ & 0 & $1(9)$ & 0 & $2(7)$ & $1(3)$ & $2(4)$ & $2(3)$ \\
\hline Deaths & 0 & 0 & 0 & 0 & 0 & 0 & 0 \\
\hline
\end{tabular}

Values are presented as number (\%).

Intent-to-treat $(I T)=$ patients who showed response to vedolizumab at 6 weeks and were randomized as part of the double-blind maintenance phase (maintenance IT population); Non-IT placebo = patients that were randomized to placebo during the induction phase and continued to received doubleblind placebo during maintenance phase (maintenance safety population only); Non-IT vedolizumab $q 4 \mathrm{w}=$ patients that did not show response to vedoli zumab at 6 weeks and received open-label vedolizumab during the maintenance phase (maintenance safety population only); Placebo combined =all patients that received placebo during the maintenance phase; Vedolizumab combined=all patients that received vedolizumab during the maintenance phase.

${ }^{a}$ Data for the ITT population.

${ }^{b}$ Patient numbers do not exactly match those shown in disposition (Fig. 1) because those patients who were discontinued from the study during the induction phase continued to be included in the safety population and have been counted within these groups.

$q 4 w_{1}$ every 4 weeks; $q 8 w_{1}$ every 8 weeks; $A E$, adverse event; SAE, serious AE. 
Supplementary Table 3. Key Safety Results in the Non-Asian Countries Subgroup of GEMINI 1 Patients: Induction Phase

\begin{tabular}{|c|c|c|c|c|}
\hline Parameter & Placebo $^{a}$ & $\begin{array}{l}\text { Vedolizumab } \\
\text { (cohort 1) }^{\mathrm{a}}\end{array}$ & $\begin{array}{c}\text { Vedolizumab } \\
\text { (cohort 2) }\end{array}$ & $\begin{array}{c}\text { Vedolizumab } \\
\text { (combined) }\end{array}$ \\
\hline No. & 120 & 196 & 466 & 662 \\
\hline Any AE & $59(49)$ & $84(43)$ & $223(48)$ & $307(46)$ \\
\hline Drug-related AE & $21(18)$ & $32(16)$ & $96(21)$ & $128(19)$ \\
\hline AE resulting in study discontinuation & $3(3)$ & 0 & $8(2)$ & $8(1)$ \\
\hline SAE & $7(6)$ & $5(3)$ & $19(4)$ & $24(4)$ \\
\hline Serious infection AE & $3(3)$ & $1(<1)$ & $3(<1)$ & $4(<1)$ \\
\hline Drug-related SAE & $2(2)$ & $1(<1)$ & $3(<1)$ & $4(<1)$ \\
\hline Serious AE resulting in study discontinuation & $3(3)$ & 0 & $6(1)$ & $6(<1)$ \\
\hline Deaths & 0 & 0 & $1(<1)$ & $1(<1)$ \\
\hline
\end{tabular}

Values are presented as number (\%).

Placebo and vedolizumab (cohort 1)=the groups that were part of the double-blind induction phase (induction intent-to-treat (ITT) population); Vedolizumab (cohort 2)=additional patients were enrolled to meet the maintenance phase sample size requirements and received open-label vedolizumab (induction safety population only); Vedolizumab combined = all patients that received vedolizumab during the induction phase. ${ }^{2}$ Data for the ITT population.

$A E$, adverse event; $S A E$, serious $A E$. 
Supplementary Table 4. Key Safety Results in the Non-Asian Countries Subgroup of GEMINI 1 Patients: Maintenance Phase

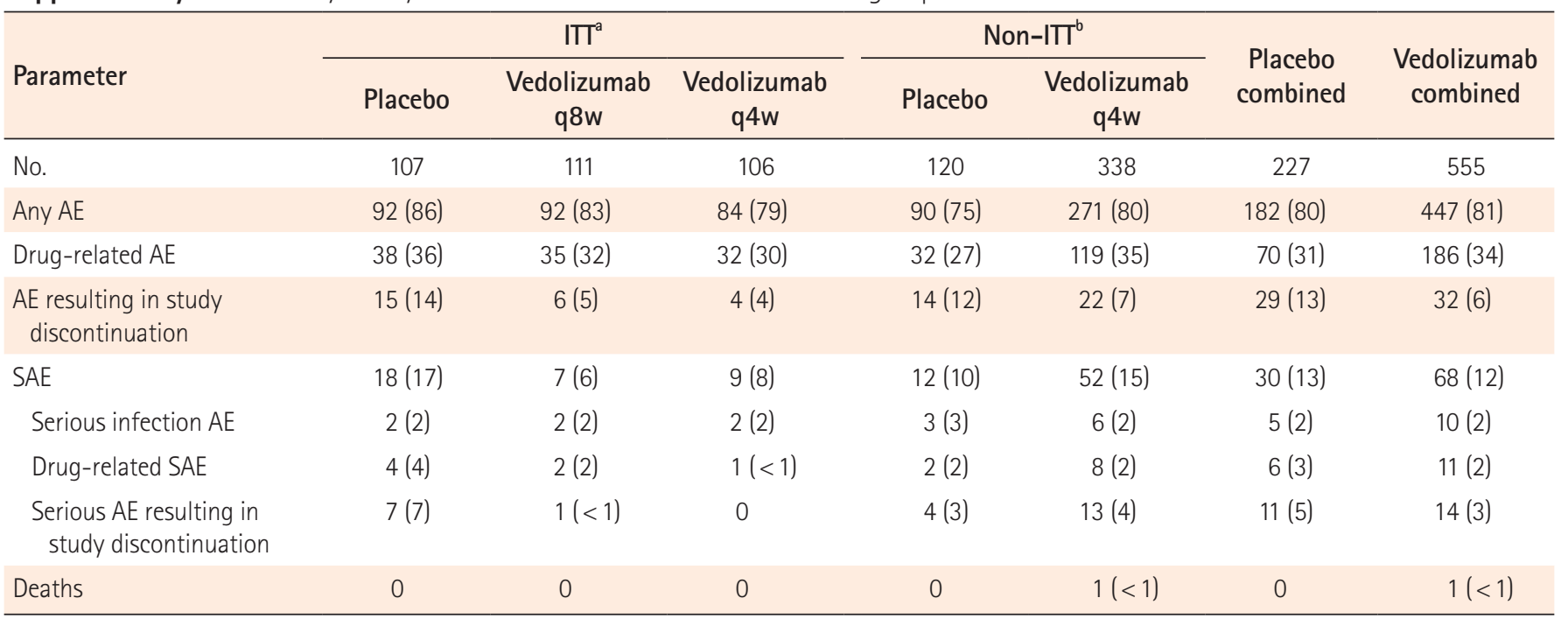

Values are presented as number (\%).

Intent-to-treat $(I T T)$ = patients who showed response to vedolizumab at 6 weeks and were randomized as part of the double-blind maintenance phase (maintenance IT population); Non-ITT placebo = patients that were randomized to placebo during the induction phase and continued to received double-blind placebo during maintenance phase (maintenance safety population only); Non-IT vedolizumab q4w = patients that did not show response to vedolizumab at 6 weeks and received open-label vedolizumab during the maintenance phase (maintenance safety population only); Placebo combined $=$ all patients that received placebo during the maintenance phase; Vedolizumab combined = all patients that received vedolizumab during the maintenance phase.

${ }^{a}$ Data for the ITT population.

${ }^{b}$ Patient numbers do not exactly match those shown in disposition (Fig. 1) because those patients who were discontinued from the study during the induction phase continued to be included in the safety population and have been counted within these groups.

$q 4 w_{\text {, every }} 4$ weeks; $q 8 w_{\text {, every } 8}$ weeks; $A E$, adverse event; SAE, serious $A E$. 